\title{
377.
}

\section{NOTE SUR LA CORRESPONDANCE DE DEUX POINTS SUR UNE COURBE.}

[From the Comptes Rendus de l'Académie des Sciences de Paris, tom. LxII. (JanvierJuin, 1866), pp. 586-590.]

Dans la théorie à laquelle se rapporte cette Note, un point de rebroussement, s'il était nécessaire d'en parler, serait censé un cas particulier du point double; mais, pour simplifier, je ne ferai attention qu'aux courbes sans point de rebroussement.

Une courbe de l'ordre $m$ peut avoir au plus $\frac{1}{2}(m-1)(m-2)$ points doubles; la différence entre ce nombre et le nombre actuel $\delta$ des points doubles d'une courbe donnée, savoir le nombre

$$
D=\frac{1}{2}(m-1)(m-2)-\delta
$$

que je nomme le défaut (en anglais, deficiency), joue, comme on sait, un rôle important dans la théorie de la courbe. En particulier, pour une courbe de l'ordre $m$ avec le défaut $D=0$, ou, comme je dis, pour une courbe unicursale de l'ordre $m$, les coordonnées $(x, y, z)$ d'un point quelconque de la courbe (je me sers toujours des coordonnées homogènes) sont proportionnelles à des fonctions rationnelles et entières du degré $m$ d'un paramètre variable $\theta$.

Cela étant, le théorème de M. Chasles: "Lorsque sur une droite deux séries de points $P, P^{\prime}$ se correspondent de manière qu'à un point donné $P$ correspondent $\alpha$ points $P^{\prime}$, et qu'à un point donné $P^{\prime}$ correspondent $\alpha^{\prime}$ points $P$, alors le nombre des points $P$ qui coïncident avec les points correspondants $P^{\prime}$ est $\alpha+\alpha^{\prime}$;" ce théorème, dis-je, s'étend sans changement à des points correspondants situés sur une courbe unicursale quelconque; et l'on peut énoncer le théorème comme il suit:

Lorsque, sur une courbe unicursale, il y a deux séries de points qui ont une correspondance $\left(\alpha, \alpha^{\prime}\right)$, le nombre des points unis est $\alpha+\alpha^{\prime}$. 
Cela donne lieu au théorème: "Lorsque, sur une courbe, avec le défaut $D$, il y a deux séries de points qui ont une correspondance $\left(x, \alpha^{\prime}\right)$, le nombre des points unis est $\alpha+\alpha^{\prime}+2 k D$," où $2 k$ est un facteur qu'il s'agit de déterminer. Cela peut se faire, sinon toujours, au moins dans la plupart des cas, au moyen du théorème que voici, tiré d'une induction qui me paraît suffisante:

En considérant sur la courbe $U=0$ un point donné $P^{\prime}$, et puis les intersections de la courbe $U=0$ par une courbe $\Theta=0$ dont l'équation contient d'une manière quelconque les coordonnées $\left(x^{\prime}, y^{\prime}, z^{\prime}\right)$ du point donné $P^{\prime}$; s'il y a $k$ intersections qui coincident avec le point $P^{\prime}$, et que les autres intersections forment un système de points $P$ qui correspondent au point donné $P^{\prime}$, et si cette correspondance est une correspondance $\left(\alpha, \alpha^{\prime}\right)$, alors le nombre des points unis est $\alpha+\alpha^{\prime}+2 k D$.

Je donne quatre exemples de ce théorème:

$1^{\circ}$. Recherche de la classe.-Si les points correspondants $P, P^{\prime}$ sont situés en ligne droite avec un point donné $O$, alors les points unis sont les points de contact des tangentes menées par le point $O$; donc le nombre des points unis est égal à la classe de la courbe. La courbe $\Theta=0$ est ici la droite $O P^{\prime}$, il y a donc une seule intersection $P^{\prime}$; donc $k=1$, et nous avons entre les points $P, P^{\prime}$ une correspondance $(m-1, m-1)$. Donc nous avons pour la classe $M$ de la courbe l'expression

$$
M=2(m-1)+2 D,
$$

où, en substituant pour $D$ la valeur

$$
D=\frac{1}{2}(m-1)(m-2)-\delta,
$$

nous trouvons

$$
M=m^{2}-m-2 \delta,
$$

comme cela doit être.

$2^{\circ}$. Recherche $d u$ nombre des inflexions. $-\mathrm{Si}$ les points $P$ sont les points de rencontre avec la courbe de la tangente au point $P^{\prime}$, alors les points unis seront les points d'inflexion. La courbe $\Theta=0$ est ici la tangente au point $P^{\prime}$; il y a ainsi deux intersections au point $P$; donc $k=2$; de plus, à chaque point $P^{\prime}$ correspondent $(m-2)$ points $P$, et à chaque point $P$ correspondent $M-2$ points $P^{\prime}$. On a donc pour le nombre des inflexions

$$
i=(m+M-4)+4 D,
$$

ou, en substituant pour $M, D$, leurs valeurs,

ce qui est juste.

$$
i=3 m(m-2)-6 \delta,
$$

Avant d'aller plus loin, il convient de généraliser le théorème, en remarquant que les intersections des courbes $U=0, \Theta=0$ peuvent former plusieurs systèmes simples ou multiples de points: les intersections peuvent être le point $P^{\prime}$ ( $k$ fois), un système de points $P$ ( $p$ fois), un système de points $Q$ ( $q$ fois), etc. Cela étant, s'il y a entre 
les points $P^{\prime}$ et $P$ une correspondance $\left(\alpha, \alpha^{\prime}\right)$, et si le nombre des points unis de ce système est $a$; s'il y a entre les points $P^{\prime}$ et $Q$ une correspondance $\left(\beta, \beta^{\prime}\right)$, et si le nombre des points unis de ce système est $b$, et ainsi de suite; alors le théorème prend la forme

$$
p \mathrm{a}+q \mathrm{~b}+\ldots=p\left(\alpha+\alpha^{\prime}\right)+q\left(\beta+\beta^{\prime}\right)+\ldots+2 k D ;
$$

c'est la forme applicable à l'exemple qui suit.

$3^{\circ}$. Recherche $d u$ nombre des tangentes doubles.-Prenons pour la courbe $\Theta=0$ le système des $(M-2)$ tangentes menées à la courbe par le point donné $P^{\prime}$; on a ici les points $P$ qui sont les points de contact de ces tangentes, et les points $Q$ qui sont les autres intersections de la courbe par ces tangentes; les intersections sont le point $P^{\prime}(M-2)$ fois (donc $\left.k=M-2\right)$, le système des points $P$ (2 fois) et le système des points $Q$ ( 1 fois). Le système $P, P^{\prime}$ est précisément celui qui donne les points d'inflexion. On a donc

$$
\alpha=\alpha^{\prime}=m-1 ;
$$

a est égal au nombre de points d'inflexion (mais, pour plus de commodité, je retiens le symbole a); $p=2$. Le système $P, Q$ est un système qui a pour points unis les points de contact des tangentes doubles, le nombre $b$ des points unis sera done $2 \tau$, en dénotant par $\tau$ le nombre des tangentes doubles. On a pour la correspondance $\left(\beta, \beta^{\prime}\right)$ entre les points $P^{\prime}$ et $Q$

enfin

$$
\beta=\beta^{\prime}=(m-3)(M-2) ;
$$

Le théorème donne ainsi

$$
q=1 \text {. }
$$

$$
2 \mathrm{a}+\mathrm{b}=2(m+M-4)+2(m-3)(M-2)+2(M-2) D ;
$$

mais nous avons ci-dessus trouvé

done enfin

$$
a=(m+M-4)+4 D
$$

$$
\mathrm{b}=2 \tau=2(m-3)(M-2)+2(M-6) D,
$$

où, en substituant pour $M$ et $D$ leurs valeurs, on retrouve la formule ordinaire

$$
2 \tau=m(m-2)\left(m^{2}-9\right)-\left(m^{2}-m-6\right) 4 x+4 x(x-1) .
$$

Parmi les intersections des courbes $U=0, \Theta=0$, il peut y avoir un système simple ou multiple de points fixes, c'est-à-dire indépendants de la position du point $P^{\prime}$; disons un système de $\lambda$ points $A$ ( $l$ fois). Il y aura dans ce cas, entre les points $P^{\prime}, A$, une correspondance $(0, \lambda)$, et les points unis du système sont les points $A$ mêmes; le nombre des points unis est donc $\lambda$; les deux côtés de l'équation contiendront les termes égaux $l \lambda$ et $l(0+\lambda)$ respectivement, qui se détruisent, ce qui fait voir qu'il est permis de négliger les points fixes $A$, et ne faire attention qu'aux points d'intersection variables. 

simple

Il est assez remarquable que le théorème général peut s'écrire sous cette forme plus

$$
p \mathrm{a}+q \mathrm{~b}+\ldots=p\left(\alpha+\alpha^{\prime}\right)+q\left(\beta+\beta^{\prime}\right)+\ldots,
$$

en comprenant parmi les systèmes formés par les intersections des courbes $U=0$, $\Theta=0$, le système du point $P^{\prime}$ ( $k$ fois), et en posant pour ce système

$$
a=0, \quad \alpha=\alpha^{\prime}=D ;
$$

le système du point $P$ ( $k$ fois) donne ainsi un terme $=0$ au côté gauche, un terme $=2 k D$ au côté droit de l'équation.

Comme dernier exemple appartenant à la formule simple

$$
a=\alpha+\alpha^{\prime}+2 k D,
$$

je prends:

4. Recherche du nombre des points sextactiques, c'est-à-dire des points qui sont tels, que par chacun passe une conique qui a dans ce point un contact du cinquième ordre avec la courbe.-Il faut prendre pour les points $P$ les intersections avec la courbe de la conique qui a au point $P^{\prime}$ un contact du quatrième ordre; les points unis seront ceux dont il s'agit. La courbe $\Theta=0$ est la conique qui a au paint $P^{\prime}$ un contact du quatrième ordre. On a ainsi, parmi les intersections, le point $P^{\prime} 5$ fois; donc $k=5$. A chaque point $P^{\prime}$ correspondent $2 m-5$ points $P$; à chaque point $P,\left(10 m^{2}-20 m-5-20 \delta\right)$ points $P^{\prime}$ (j'emprunte le terme $-20 \delta$ d'une formule que vient de donner M. Zeuthen); donc la formule donne pour le nombre des points unis

$$
10 m^{2}-18 m-10-20 \delta+10 D
$$

c'est-à-dire

$$
15 m^{2}-33 m-30 \delta .
$$

Mais cette expression comprend le nombre $3 m(m-2)-6 \delta$ des inflexions; en effet, pour un point d'inflexion, la conique avec contact du quatrième ordre se réduit à la tangente prise deux fois, ce qui est une conique avec contact du cinquième ordre. Donc enfin le nombre des points sextactiques sera

$$
m(12 m-27)-24 \delta,
$$

ou, pour une courbe sans points doubles,

$$
m(12 m-27) \text { : }
$$

ce qui s'accorde avec la valeur que j'ai trouvée par d'autres moyens, [341]. 